\title{
Platin-Kohle-Abdruck-Technik angewandt auf den Feinbau der Milchröhren
}

\section{Doctoral Thesis}

\section{Author(s):}

Moor, Hans Jakob

Publication date:

1959

Permanent link:

https://doi.org/10.3929/ethz-a-000090885

Rights / license:

In Copyright - Non-Commercial Use Permitted 
Prom.-Nr. 2872

\title{
Platin-Kohle-Abdruck-Technik angewandt auf den Feinbau der Milchröhren
}

\author{
Von der \\ EIDGENÖSSISCHEN \\ TECHNISCHEN HOCHSCHULE IN ZÜRICH \\ zur Erlangung der Würde eines Doktors \\ der Naturwissenschaften \\ genehmigte \\ PROMOTIONSARBEIT \\ vorgelegt von \\ HANS MOOR \\ dipl. Natw. E.T.H. \\ von Baselstadt
}

Referent: Herr Prof: Dr. A. FReY-Wys LING

Korreferent: Herr Prof. Dr. K. MühLETHALER 


\title{
Platin-Kohle-Abdruck-Technik angewandt auf den Feinbau der Milchröhren ${ }^{1}$
}

\author{
H. MoOR \\ Laboratorium für Elektronenmikroskopie, Institut für Allgemeine Botanik der Eidgenös- \\ sischen Technischen Hochschule, Zürich
}

Eingegangen am 12. Januar 1959

\begin{abstract}
Das Auflösungsvermögen von vorbeschatteten Abdrucken wird durch die Schichtdicke, die Eigenstruktur und die Dichte des Beschattungsmaterials bestimmt. Die Eigenschaften des kontrastreichen, schwerlöslichen Platins als Beschattungsmaterial können durch Beimischung von Chrom oder Kohle bis zu einem gewissen Grade verbessert werden. Die praktisch erzielten Auflösungen von $30-40 \AA$ entsprechen den theoretisch zu erwartenden. Durch indirekte Aufdampfung der Kohle ist es möglich, dünnste Trägerfilme von genügender Tragfähigkeit herzustellen; ihre Schichtdicke wurde mit verschiedenen, voneinander unabhängigen Methoden bestimmt.

Die Entwicklung der Milchröhren von Euphorbia splendens erfolgt durch ,,interzellulares Schlauchwachstum". Die Zellwand entsteht allein durch Apposition als ,Multi-net-growth“. Sie ist analog den Verhältnissen in Faserzellen und Tracheiden in eine Primärwand, Übergangslamelle und Sekundärwand gegliedert; eine Tertiärlamelle fehlt. Die Primärwand weist Folientextur auf, die Übergangslamelle ein in zwei Richtungen überkreuzt verwobenes Fibrillensystem, die Sekundärwand einen 50- bis 100-schichtigen Lamellenkomplex mit Schraubentextur, in welchen der Umlaufsinn der Schraube von einer Schicht zur andern wechselt. Die Gleichzeitigkeit von Flächen- und Dickenwachstum wird einerseits durch die in der Folge der Lamellen netzartig erscheinende Textur der Zellulose und anderseits durch den hohen Gehalt an leicht hydrolysierbarem Pektin ermöglicht.
\end{abstract}

Die elektronenmikroskopische Untersuchung pflanzlicher Zellwände stellt vor allem präparationstechnische Probleme. Die übliche Einbettungs- und Schneidetechnik liefert aus verschiedenen Grïnden nur unzureichende Resultate: Erstens schrumpfen

${ }_{1}$ Die vorliegende Arbeit wurde im Laboratorium für Elektronenmikroskopie des Institutes für Allgemeine Botanik der Eidgenössischen Technischen Hochschule in Zürich durchgeführt. Den Vorstehern, Herrn Prof. Dr. A. Frey-Wyssling und Herrn Prof. Dr. K. Mühlethaler, meinen geschätzten Lehrern, möchte ich für ihre Unterstützung und Förderung meinen besten Dank aussprechen. Ebenso bin ich den Herren Dr. A. Vogel und Dr. H. R. Müller für wertvolle Anregungen zu Dank verpflichtet. 